\title{
The Interaction of Bilayer Graphene with an External Magnetic Field
}

\author{
Yuri Petrovich Rybakov ${ }^{1}$, Abdullahi Bappah Ahmed ${ }^{2, *}$ \\ ${ }^{1}$ Department of Theoretical Physics and Mechanics, Faculty of Science, Peoples' Friendship University of Russia, Moscow, Russia \\ ${ }^{2}$ Department of Physics, Faculty of Science, Gombe State University, Gombe, Nigeria
}

Email address:

garkuwaz@yahoo.com (A. B. Ahmed)

\section{To cite this article:}

Yuri Petrovich Rybakov, Abdullahi Bappah Ahmed. The Interaction of Bilayer Graphene with an External Magnetic Field. International Journal of Applied Mathematics and Theoretical Physics. Vol. 3, No. 3, 2017, pp. 74-77. doi: 10.11648/j.ijamtp.20170303.15

Received: April 14, 2017; Accepted: May 19, 2017; Published: June 16, 2017

\begin{abstract}
In this phenomenological approach to the study of magnetism in bilayer graphene, the chiral model of graphene was employ to describe the interaction of the bilayer graphene with an external magnetic field. The simplest scalar chiral model of graphene suggested earlier and based on the SU (2) order parameter is generalized by including 8-spinor field as an additional order parameter for the description of spin (magnetic) excitations in the bilayer graphene. As an illustration we study the interaction of the bilayer graphene with the external magnetic field orthogonal to the plane. The Lagrangian density of the model was constructed; The Lagrangian density of the model includes the three interacting terms, the spinor field, chiral field, and the electromagnetic field. The domain wall solution describing the bilayer graphene configuration is introduced for studying the magnetic field behavior in the central domain of the material; the solution to the inhomogeneous equations were found using the Green's function method, at small radial field, the paramagnetic behavior of the material was revealed and the strengthening of the magnetic intensity inside the material in the central domain of the material was also revealed.
\end{abstract}

Keywords: Bilayer Graphene, Chiral Model, Domain Wall, 8-Spinor, Green Function, Bessel's Function

\section{Introduction}

Since the very discovery of this novel material it has attracted deep interest of researchers (theoretical and experimental) due to its extraordinary properties [1]. Graphene is a monolayer of carbon atoms packed into a dense honeycomb crystal structure, which can be obtained by mechanical exfoliation [2]. The electronic properties of graphene; a two dimensional crystal of carbon atoms are exceptionally novel [3]. Carbon atoms have a unique capability of associating with each other in different ways at the macro and nanoscopic scales to form various structures which turns to be unique [4] Bilayer graphene which is one of them, consists of two coupled mono-layers of carbon atoms, each with a honeycomb crystal structure [5]. As there are two layers, bilayer graphene represents the thinnest possible limit of an intercalated material,in monolayer graphene, each unit cell containing two carbon atoms, labeled $\mathrm{A}$ and B. Bilayer graphene consists of two coupled monolayer's, with four atoms in the unit cell, labeled A1, B1 on the lower layer and A2, B2 on the upper layer, the layers are arranged so that one of the atoms from the lower layer B1 is directly below an atom, A2, from the upper layer [6]. There are two stacking arrangement of the bilayer graphene, depending on the orientation of the mono-layers:

a AA stacking

b AB Bernal stacking

These two possibilities can be seen in Figure1, in this study the AB (Bernal) stacking was adopted, as it's known to be the most stable structure of bilayer graphene [7]. However magnetism of conventional metal is composed of two different contributions, the Pauli paramagnetism due to the spin magnetic moment, and the Landau diamagnetism which is due to the orbital motion of the electrons.

One of the present obstacles of graphene despite having novel properties is the absence magnetism; therefore most researchers in graphene base material mainly pay attention on the substantial magnetism in graphene. The incorporation of magnetism to the long list of graphene capabilities has been pursued since its first isolation in 2004 by Geim and Novoselov; it therefore motivates us to undergo this study. The chiral model of graphene is been adopted; the model was recently developed by Rybakov.Yu.P in 2012 to add to the 
existing condensed matter field theoretical models $[8,9$, and 10].

Furthermore, the choice for the model follows the fact that electrons in graphene show relativistic behavior, and the system is therefore an ideal candidate for the test of quantumfield theoretical models. "Electrons travel through it so fast that their behavior is governed by the theory of relativity rather than classical physics" (The Economics, 2006). Although the electrons in graphene are conferred to move on a plane, the electromagnetic field through which they interact extends throughout the 3D space [11]. Another important observation is that the electron mobility in graphene is about ten times higher than the mobility of commercial silicon wafers and electrons can travel huge distances $(300 \mathrm{~nm}$ or more) without being scattered [12]

In this phenomenological approach to the description of magnetism in bilayer graphene, the action of the external magnetic field orthogonal to the bilayer graphene planes was investigate, considering the fact that magnetism in graphene and other carbon structures prove to be extremely anisotropic [13].

\section{Chiral Model}

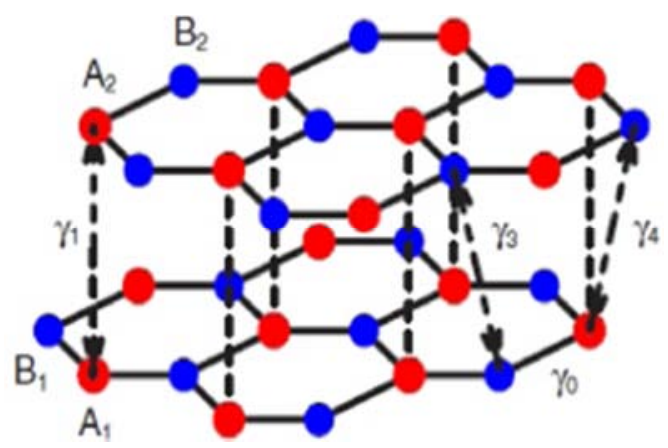

Figure 1. Stacking of bilayer graphene, consisting of two coupled monolayers of graphene: (a) AA stacking; (b) AB Bernal stacking. Each layer consists of two in equivalent site $A$ and $B$, the intra-layer and intra-layer hoping integrals are $\gamma_{0}$ and $\gamma_{1}$ respectively(Barlas, Yang, \& MacDonald, 2012).

The simplest scalar chiral model is based on the so-called $s p$-hybridization effect of the carbon atom valence electrons. In the honeycomb hexagonal mono-layer lattice three of the valence electrons form strong covalent bonds with the neighbors and the fourth electron is "free", its state being linear combination of $s$ - and $p$-states. In the scalar model the $s$-state is described by the scalar field $a_{0}$ and the $p$-state - by the vector field $\vec{a}$. These two fields are combined in the SU (2) matrix $U=a_{0} \tau_{0}+i(\vec{a} \vec{\tau})$ where $\tau_{0}, \vec{\tau}$ denote the unit and the Pauli matrices respectively and the normalization condition $a_{0}^{2}+\vec{a}^{2}=1$ is imposed [1]. Spin excitations in the model are described by the two Dirac spinors $\psi_{1}, \psi_{2}$ corresponding to the electrons in the two independent triangular sub-lattices of the graphene (so-called quasi-spin excitations). It is convenient to introduce the combined field $\psi=\xi \otimes\left(\psi_{1} \oplus \psi_{2}\right)$ as the new order parameter of the model, where $\xi$ stands for the first column of the $U$-matrix. The Lagrangian density of the model reads:

$$
L=\frac{I}{2} \bar{D}_{\mu} \bar{\psi} P D^{\mu} \psi-\frac{\lambda^{2}}{2} \vec{a}^{2} j_{\mu} j^{\mu}+i \mu_{0} \vec{a}^{2} \bar{\psi} \sigma_{\mu \nu} F^{\mu \nu} \psi
$$

Here the interaction with the electromagnetic field is introduced via the extension of the derivative: $D_{\mu}=\partial_{\mu}-$ $i e_{0} A_{\mu} \Gamma_{\mathrm{e}}$ where $\Gamma_{e}=\left(1-\tau_{3}\right) / 2$ stands for the charge operator corresponding to the natural boundary condition $a_{0}(\infty)=1$ and $P=\gamma^{\mu} j_{\mu}$ is the projector on the positive energy states. The model uses the Dirac currents $j_{\mu}=\bar{\psi} \gamma_{\mu} \psi$ and also the direct Pauli interaction: $\sigma_{\mu \nu} F^{\mu \nu}=\left[\gamma_{\mu}, \gamma_{v}\right]\left(\partial^{\mu} A^{v}-\partial^{v} A^{\mu}\right) / 4$, the parameter $I$ corresponds to the exchange energy per lattice spacing and $\mu_{0}$ is the Bohr magneton per spacing cubed.

As an illustration let us study the case with the orientation of the magnetic field $\vec{B}_{0}$ along the $z$ axis using the cylindrical coordinates $\rho, \phi, z$, and also introduce the vector potential $A_{\phi}=A$, with the intensity of the magnetic field being $B_{z}=\partial_{\rho}(\rho A) / \rho, B_{\rho}=-\partial_{z} A$, and the natural boundary condition at infinity being imposed: $A(z \rightarrow \infty)=$ $B_{0} \rho / 2$.

The model in question admits the evident symmetry $\psi_{1} \Leftrightarrow \psi_{2}$ considering the infinite carbon plane and $\gamma_{0}$ invariance $\psi \Rightarrow \gamma_{0} \psi$. Where $Z=-Z$ corresponds to the reflection symmetry considering the 2 planes .That permits one to introduce 2-spinor $\varphi$ where $\psi_{1}=\psi_{2}=\operatorname{col}(\varphi, \varphi), \varphi=\operatorname{col}(w, u)$. To simplify the calculations, we us suppose the smallness of the radial magnetic field: $B_{\rho} \ll B_{z}$.

In this approximation the new discrete symmetry holds $\varphi \Rightarrow-\sigma_{3} \varphi, w \Rightarrow-w, u \Rightarrow u^{*}, a_{2,3} \Rightarrow-a_{2,3}$, that permits one to introduce the chiral angle? ?: $a_{0}=\cos \Theta, a_{1}=\sin \Theta$ and consider the axially symmetric configuration: $w=$ $0, u=u(\rho, z), \Theta=\Theta(\rho, z)$.

\section{The Lagrangian Density}

The Lagrangian density $(L)$ of the model includes the three interacting fields the spinor field, chiral field, and the electromagnetic field. As a result the Lagrangian density takes the form:

$$
L=-8 I\left[R^{2}\left(\partial_{\perp} \Theta\right)^{2}+\frac{1}{4}\left(\partial_{\perp} R\right)^{2}+e_{0}^{2} R^{2} A^{2} \sin ^{2} \Theta\right]+8 \mu_{0} \operatorname{Rin}^{2} \Theta B-\frac{\mathrm{A}^{\prime 2}}{8 \pi}-8 \lambda^{2} R^{2} \sin ^{2} \Theta
$$

The model contains the two constant parameters: the exchange energy $I$ per lattice spacing and some characteristic inverse length $\sqrt{\lambda}$. Where $\mu_{0}>0$ denotes the Bohr magneton per lattice spacing cubed.
The new variable for our spinor field is introduced: $R=u^{2}$ and $\partial_{\perp}$ signifies the differentiation with respect to $\rho$ and $z$. The equations of motion corresponding to the three interacting fields; the spinor $(R)$, chiral (??) and 
electromagnetic field $(A)$ in (1) read as follows:

$$
\begin{gathered}
I\left[\nabla^{2} R-4 R(\nabla \Theta)^{2}-4 e_{0}^{2} R A^{2} \sin ^{2} \Theta\right]=2 \sin ^{2} \Theta\left[2 \lambda^{2} R-\mu_{0} B\right] \\
I\left[2 \nabla(\nabla \Theta) R^{2}-e_{0}^{2} R^{2} A^{2} \sin 2 \Theta\right]=R \sin 2 \Theta\left[\lambda^{2} R-\mu_{0} B\right] \\
\frac{1}{4 \pi} \nabla^{2} A=16 I e_{0}^{2} R^{2} A \sin ^{2} \Theta+8 \mu_{0} \partial_{\rho}\left(R \sin ^{2} \Theta\right) .
\end{gathered}
$$

Let us now search for the solution to the equations (2), (3), (4) in the asymptotic domain $z \rightarrow \infty$, where, $\Theta \rightarrow 0$, $R=1 / 4+\xi, \quad A=B_{0} \rho / 2+\beta, \quad \xi \rightarrow 0, \beta \rightarrow 0 . \quad$ thus, the equation for our chiral angle in (3) takes the form:

$$
I\left[\nabla^{2} \Theta-\frac{1}{4} e_{0}^{2} B_{0}^{2} \rho^{2} \Theta\right]=\Theta\left(\lambda^{2}-4 \mu_{0} B_{0}\right)
$$$$
\xi=\Theta_{0}^{2} \exp \left(-2 v \rho^{2}-2 k z\right) N(\rho) ; \beta=\delta \Theta_{0}^{2} \exp \left(-2 v \rho^{2}-2 k z\right) K(\rho)
$$

where the radial functions $\mathrm{N}(\rho)$ and $\mathrm{K}(\rho)$ satisfy the following equations:

$$
N^{\prime \prime}+N^{\prime}\left(\frac{1}{\rho}-8 v \rho\right)+N\left[2 B_{0}\left(e_{0}-8 \frac{\mu_{0}}{I}\right)+4 \frac{\lambda^{2}}{I}+e_{0}^{2} B_{0}^{2} \rho^{2}\right]=\frac{1}{2} e_{0}^{2} B_{0}^{2} \rho^{2}+e_{0} B_{0}+\frac{2}{I}\left(\lambda^{2}-3 \mu_{0} B_{0}\right)
$$$$
K^{\prime \prime}+K^{\prime}\left(\frac{1}{\rho}-8 v \rho\right)+K\left(4 k^{2}-8 v \rho^{2}-\frac{1}{\rho^{2}}\right)=\rho .
$$

Let us now estimate the magnetic intensity:

$$
B_{z}=B_{0}+b_{z}, b_{z}=\frac{1}{\rho} \partial_{\rho}(\rho \beta), B_{\rho}=b_{\rho}=-\partial_{z} \beta
$$

However, at small $\rho \rightarrow 0$ one finds from (10) that $K \approx$ $\rho^{3} / 8$, and therefore the intensity of the magnetic field reads:

$$
\begin{aligned}
& b_{z}=\pi e_{0} B_{0}\left(e_{0} I-4 \mu_{0}\right) \Theta_{0}^{2} \rho^{2} \exp \left(-2 v \rho^{2}-2 k z\right) \\
& b_{\rho}=\frac{\pi k}{2} e_{0} B_{0}\left(e_{0} I-4 \mu_{0}\right) \Theta_{0}^{2} \rho^{3} \exp \left(-2 v \rho^{2}-2 k z\right)
\end{aligned}
$$

As can be seen from the equations (11) and (12), according to the sign of the multiplier $e_{0} I-4 \mu_{0}$ our graphene material reveals diamagnetic or paramagnetic behavior. Therefore, it should be interesting to obtain numerical estimates for the parameters of the model. In view of the definitions adopted one has

$$
e_{0}=\frac{e}{\hbar c}, \quad \mu_{0}=\frac{e \hbar}{2 m_{e} c a^{3}}, \quad I=\frac{\mathrm{E}_{\mathrm{exch}}}{\mathrm{a}} .
$$

Where, the exchange energy is usually adopted as $E_{\text {exch }}=$ $2.9 \mathrm{eV}$ and the lattice spacing as $a=3.56 \cdot 10^{-8} \mathrm{~cm}$, with $e$ being the absolute value of the electron charge. Finally, one can find the following numerical values:

$$
e_{0} I=2 \cdot 10^{3} \text { Gauss, } \mu_{0}=2 \cdot 10^{2} \text { Gauss. }
$$

It means that the parameter $e_{0} I-4 \mu_{0}$ is positive and the weakening of the magnetic field inside the bilayer graphene is predicted and it's strengthening for small $\mathrm{r}$ in accordance with (12) and (13). In view of the importance of the latter conclusion, it would be desirable to investigate the magnetic field behavior in the central domain of the bilayer graphene material.

\section{Domain Wall Structure}

In view of the importance of the later conclusion it would desirable to investigate the magnetic field behavior in the central domain of the bilayer graphene, i.e. at small $r$ but arbitrary to $z$. To this end, we consider the extrapolation of the configuration (6) to the domain wall structure of the form:

$$
\begin{gathered}
\theta=4 \arctan \left[\exp \left(-v \rho^{2}-k z\right)\right] \\
\partial_{\rho} \theta=-4 v \rho \frac{1}{\cosh \left(v \rho^{2}+k z\right)} \\
\partial_{z} \theta=-2 k \frac{1}{\cosh \left(v \rho^{2}+k z\right)} \\
\sin ^{2} \theta=4 \frac{\tanh ^{2}\left(v \rho^{2}+k z\right)}{\cosh ^{2}\left(v \rho^{2}+k z\right)}
\end{gathered}
$$

By inserting equation (16) and $A=B_{0} \rho / 2+\beta, R \approx 1 / 4$ into (4), would amount to the following inhomogeneous equation:

$$
\nabla^{2} \beta-\frac{\beta}{\rho^{2}}=2 \pi \rho I B_{0} e_{0}^{2} \sin ^{2} \theta+8 \pi \mu_{0} \partial_{\rho} \sin ^{2} \theta \equiv 2 \pi \rho j
$$

The solution to the equation (20) satisfying boundary condition $\beta(\rho=0)=0$ can be obtained by the method of Green's function: 


$$
\beta=\int_{-\infty}^{\infty} d z^{\prime} \int_{-\infty}^{\infty} d s \exp \left[i s\left(z-z^{\prime}\right)\right] \int_{0}^{\rho} d \rho^{\prime} \rho^{\prime} j^{\prime}\left[I_{1}(s \rho) K_{1}\left(s \rho^{\prime}\right)+K_{1}(s \rho) I_{1}\left(s \rho^{\prime}\right)\right]
$$

where $I_{1}$ and $K_{1}$ stand for the modified Bessel functions of the imaginary argument. Taking into account their asymptotic behavior as $\rho \rightarrow 0: I_{1}(\rho) \approx \rho / 2, K_{1}(\rho) \approx \rho^{-1}$

$$
\begin{gathered}
I_{1}(s \rho) K_{1}\left(s \rho^{\prime}\right)+I_{1}\left(s \rho^{\prime}\right) K_{1}(s \rho)=\frac{1}{2}\left(\frac{\rho}{\rho^{\prime}}-\frac{\rho^{\prime}}{\rho}\right) \\
\frac{1}{2} \int_{0}^{\rho} d \rho^{\prime} \rho^{\prime 2}\left(\frac{\rho}{\rho^{\prime}}-\frac{\rho^{\prime}}{\rho}\right)=\frac{1}{2}\left(\rho \cdot \frac{1}{2} \rho^{2}-\frac{1}{\rho} \cdot \frac{1}{4} \rho^{4}\right)=\frac{\rho^{3}}{8}
\end{gathered}
$$

one obtains:

$$
\beta=\frac{\pi \rho^{3} e_{0} B_{0}}{\cosh ^{2}(k z)}\left[I e_{0} \tanh ^{2}(k z)+4 \mu_{0} \tanh (k z)\left(1-2 \tanh ^{2}(k z)\right)\right]
$$

The expression (24) being positive is consistent with the numerical values in (15).Therefore, in the presence of an external magnetic field orthogonal to the planes one confirms the paramagnetic behavior of the bilayer graphene in the central domain at small $\rho$.

\section{Conclusion}

In order to study the magnetic behavior of a bilayer graphene in the presence of an external magnetic field orthogonal to the plan, the two phenomenological approaches for the description were analyzed: The 8-spinor generalization of the scalar chiral model of graphene was considered. Using some symmetry properties of the model, the gamma 5 invariance Lagrangian density quadratic in derivative was constructed using the principle of the energy positivity and that of correspondence with the scalar model was simplified and the equation of motion of the three interacting field (spinor, chiral and electromagnetic) was realized. The bilayer graphene reveals a diamagnetic or paramagnetic behavior according to the sign of the multiplier in equations (11) and (12), the magnetic field intensity at small $\rho$ base on the parameters of the model was estimated, and it was found to be positive. The scalar model admits a very simple domain wall solution describing the bilayer graphene configuration. In the case of the magnetic field orthogonal to the bilayer graphene, according to equation (17) it was also found to be positive at small $\rho$ that fact confirms the paramagnetic behavior of the bilayer graphene and the strengthening of the magnetic field intensity inside the material was revealed in the central domain. In future the effect of temperature would be considered for further investigations using the chiral model of graphene.

\section{References}

[1] Rybakov Yu P: On chiral model of graphene//Solid State Phenomena, v.190.2012, P.59-62.

[2] ERJUN KAN, ZHENYU LI and JINLONG YANG YANG MAGNETISM IN GRAPHENE SYSTEMS: NANO, 2008. Issue 06: Vol. 03.

[3] Berashevich J. [et al.] Properties of graphene: a theoretical perspective: Advances in Physics, 2010. - 4: Vol. 59.

[4] Jean Paul Issi, Paulo T. Arauja and Mildred S Dresselhaus Electron and Phonon Transport in Graphene in and out of the Bulk [Book Section] // Physics of Graphene / book auth. Aoki H. and Dresselhaus M. S.: Springer, 2014.

[5] Castro E.v. et al. Phys. Rev.lett.99,216802(2007).

[6] Santos J. M. B. Lopes dos, N. M. R. Peres and Neto A. H. Castro Graphene bilayer with a twist: electronic structure: arXiv.org > cond-mat > arXiv:0704.2128, 2007.

[7] McCann Edward and Vladimir. I. Fal'kovski Weak Localised and Spin-Orbit Coupling in Monolayer and Bilayer Graphene: Spinger, 2014.

[8] Rybakov Yu PSpin excitations in chiral model of graphene//Solid State Phenomena, v.233-234. 2015, P.16-19.

[9] Rybakov Yu P, Ahmed A. B, Iskandar M., Magnetic excitations of graphene in 8-spinor realization of chiral model// RUDN Jouranal of Mathematics, Information, Physics[Bulletin of Peoples Friendship Unoversity of Russia], vol. 25.2017 , No. 3 .

[10] Philip Kim Graphene and Relativistic Quantum Physics [Report]. - New york 10027, USA: Saminaire Pointcare, 2004.

[11] Nat'alia Menezes [et al.] Valley- and spin-splitting due to interactions in graphene [Journal]. - [s.1.]: arxiv.org/pdf/1601.07454., 2016. - Vol. 1.

[12] Erjun Kan, Zhenyu Li and Jinlong Yang Magnetism in Graphene Systems [Journal]. - [s.1.]: Nano, 2008. - 06: Vol. 03 .

[13] Yuchen Ma [et al.] Magnetic properties of vacancies in graphene//New Journal of Physics, Vol. 6. 2004, P. 68. 